\title{
This Week in The Journal
}

In honor of the 50th anniversary of the Society for Neuroscience ( $\mathrm{SfN}$ ), the editors of The Journal of Neuroscience invited some of the attendees of the very first annual meeting, as well as the intellectual descendants of attendees, to write reviews focused on developments in some of the many subfields within Neuroscience. Many of these reviews are included in this issue, and others are planned for future issues throughout the year.

Our first review is by Barry Stein (311), who presented work on "Velocity dependent directional selectivity in cat superior colliculus" at the first annual meeting of SfN. Since then, he has continued to investigate the superior colliculus, whose function, he notes, is similar to that of SfN: bringing information together from diverse sources. He reviews how studies of the superior colliculus have led to a deeper understanding of multisensory integration.

Huda Akil and Bruce McEwen also presented posters at the first annual meeting, but on different topics. Akil's poster focused on analgesia resulting from brain stimulation, whereas McEwen revealed that stress hormones from the adrenal gland bind to receptors in the hippocampus. Their work later converged, as Akil began to study stress-induced analgesia. Together, Akil and McEwen (12-21) review how our concept of stress and our understanding of how it affects emotion have changed over the past 50 years.

Darcy Kelley was a copresenter with Bruce McEwen on the poster about stress-hormone binding in the hippocampus, but she went on to study animals without a hippocampus: Xenopus frogs. Studies of vocal communication in this species have allowed Kelley and her colleagues to delve into diverse areas of neuroscience, from mapping neural circuits that control motor behavior to discovering how hormones and evolution alter these circuits to create sexand species-specific behaviors. Their review (22-36) summarizes the numerous discoveries made so far, setting the stage for more exciting work in the future.

Of course, sex differences in the brain extend far beyond the neural circuits underlying vocal communication in frogs. Indeed, the study of how sex hormones shape the brain to drive sex-specific behaviors has grown dramatically over the past few decades. Melissa Hines reviews (37-43) the major developments in this field and discusses how such findings may help us understand sex differences in the prevalence of psychiatric diseases and neurological conditions, as well as how they inform issues of gender identity.

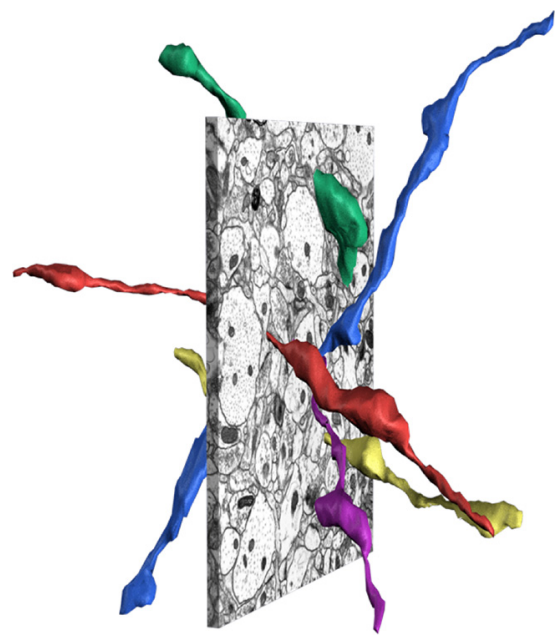

Three-dimensional reconstruction from electron micrographs of Schaffer collaterals in the hippocampus. See Harris $(60-76)$ for details.

Much of what we know about sensory processing came from studies of the visual system. Such studies were well underway at the time SfN was founded. Trenholm and Krishnaswamy surveyed vision researchers in an attempt to identify the most influential articles in the field, and in their review (44-52) they provide short synopses of these articles with reference to related work.

Neuroscientists' appreciation of the importance of the hippocampus also started to grow about a decade before SfN was founded. Laura Colgin (53-59) notes two key discoveries that were reported shortly before the first meeting: John O'Keefe's description of place cells, and Case Vanderwolf's description of EEG patterns in the hippocampus. Colgin reviews how these fields have developed since then.

The first annual meeting included a "demonstration and discussion lounge" focused on morphology and ultrastructure. Although Kristen Harris did not come onto the neuroscience scene until several years after this session, Bill Greenough, one of her early collaborators, was present. Her photoessay in this issue (6076 ) includes an image from her work with Greenough, as well as many other beautiful images of cellular ultrastructure that she has generated during her career.

Our ability to address long-standing questions in neuroscience has depended greatly on the development of new technologies. Advances over the past 5 decades have driven discoveries across all scales of inquiry, from the molecular (using technologies reviewed by Deanna Benson (77-84)) to the whole brain (using techniques reviewed by Kia Nobre and Freek van Ede (85-96)). Ongoing advances will surely continue to drive the field forward. In our final review of this issue (97-102), Cara Altimus, Bianca Marlin, and other members of the SfN Trainee Advisory Committee provide their view on what advances might occur over the next 50 years. We hope they and many readers of these articles will be around for the 100th anniversary of $\mathrm{SfN}$, to see whether these predictions come true.

This Week in The Journal was written by @Teresa Esch, Ph.D. https://doi.org/10.1523/JNEUROSCI.twij.40.1.2020. 